\title{
Développement en fraction continue à l'entier le plus proche, idéaux $\alpha$-réduits et un problème d'Eisenstein
}

\author{
par \\ Pierre Kaplan (Nancy) et Yoshio Mimura (Osaka)
}

1. Introduction. Soit $D$ un entier positif non carré congru à 1 modulo 4 . Le problème d'Eisenstein ([1], [2]) dont il sera question ici est de trouver un critère pour que l'équation

$$
X^{2}-D Y^{2}=4
$$

ait des solutions impaires, ce qui n'est possible que si $D \equiv 5(\bmod 8)$. Dans le travail [4], paru en 1990 dans cette revue, le résultat suivant a été prouvé :

THÉORÈme 0. Soient $\ell_{0}$ et $\ell_{0}^{*}$ respectivement les longueurs des périodes des développements en fraction continue des nombres $\sqrt{D}$ et $(1+\sqrt{D}) / 2$.

(a) Si l'équation (1.1) a des solutions impaires, $\ell_{0}^{*}+4 \leq \ell_{0} \leq 5 \ell_{0}^{*}$.

(b) Si l'équation (1.1) n'a pas de solution impaire, $\ell_{0}^{*} / 3 \leq \ell_{0} \leq 3 \ell_{0}^{*}-8$.

Ce résultat, qui est le meilleur possible, ne donne pas de critère. Les autres résultats connus concernant ce problème d'Eisenstein ([5-8], [11], [12]) font aussi intervenir les développements en fraction continue des nombres $\sqrt{D}$ et $(1+\sqrt{D}) / 2$.

Le but de ce travail est d'obtenir un critère en considérant, au lieu des développements en fraction continue usuels, les développements en fraction continue à l'entier le plus proche des nombres $\sqrt{D}$ et $(1+\sqrt{D}) / 2$. Le développement en fraction continue à l'entier le plus proche d'un nombre réel $\varphi$ est défini par

$$
\varphi_{0}=\varphi, \quad \varphi_{n}=q_{n}+\varepsilon_{n} / \varphi_{n+1}, \quad q_{n} \in \mathbb{Z}, \varepsilon_{n}= \pm 1, \quad \varphi_{n+1}>2,
$$

de sorte que $q_{n}$ est, pour tout $n \geq 0$, l'entier le plus proche de $\varphi_{n}$. Si $\varphi$ est un nombre irrationnel quadratique son développement est périodique à partir d'un certain rang; soient $L_{0}$ et $L_{0}^{*}$ les longueurs respectives des périodes des

1991 Mathematics Subject Classification: 11R11, 11A55. 
développements en fraction continue à l'entier le plus proche des nombres $\sqrt{D}$ et $(1+\sqrt{D}) / 2$. Nous obtenons le résultat suivant :

ThÉORÈme 1. Soit $D>5$ un entier non carré congru à 1 modulo 4.

(a) Si l'équation $X^{2}-D Y^{2}=4$ a des solutions impaires alors

$$
2 L_{0}^{*}<L_{0} \leq 4 L_{0}^{*} \text {. }
$$

(b) Si l'équation $X^{2}-D Y^{2}=4$ n'a pas de solution impaire alors

$$
L_{0}^{*} / 2 \leq L_{0}<2 L_{0}^{*} .
$$

Le Théorème 1 montre que $L_{0}^{*} / 2 \leq L_{0}<2 L_{0}^{*}$ si $D \equiv 1(\bmod 8)$ et donne un critère pour le problème d'Eisenstein quand $D \equiv 5(\bmod 8)$.

Dans la théorie du développement en fraction continue à l'entier le plus proche la notion de réduction est remplacée par celle d' $\alpha$-réduction. C'est à cette notion, en particulier à celle d'idéal $\alpha$-réduit, qu'est consacré le $\S 2$. Dans le $\S 3$ nous donnons un exposé bref mais complet de la théorie du développement en fraction continue à l'entier le plus proche des nombres quadratiques et des idéaux associés. Une partie du contenu de ce paragraphe se trouve dans le travail [4] de Hurwitz mais, plutôt que d'y référer, il nous a semblé plus simple et plus clair d'exposer les points essentiels de cette théorie peu connue. Au $\S 4$ nous définissons et étudions une application $\psi$ de l'ensemble $E$ des idéaux $\alpha$-réduits de l'ordre $O_{4 D}$ de discrimininant $4 D$ sur l'ensemble $E^{*}$ des idéaux $\alpha$-réduits de l'ordre $O_{D}$ de discriminant $D$. Soit $N=\operatorname{card}(E)$ et $N^{*}=\operatorname{card}\left(E^{*}\right)$. L'étude de $\psi$ nous permet, pour $D \equiv 5(\bmod 8)$, de déterminer $N$ en fonction du nombre des idéaux de $E^{*}$ satisfaisant à certaines conditions, obtenant, pour les idéaux $\alpha$-réduits, un résultat analogue à celui de [6], Théorème 1 , pour les idéaux réduits. Au $\S 5$ nous démontrons le Théorème $1(\mathrm{a})$ à partir des résultats du $\S 4$. Au $\S 6$ nous étudions la croissance de l'application $\psi$ quand l'idéal $\alpha$-réduit $I$ décrit sa période, et c'est le fait que cette croissance est régulière, plus régulière que la croissance de l'application analogue étudiée dans [5] pour les périodes d'idéaux réduits, qui nous permet, malgré la multiplicité des cas à envisager, de parvenir à démontrer le Théorème 1(b).

La démonstration du Théorème 1 nous fournit le résultat suivant qui permet de comparer les nombres $N$ et $N^{*}$ des idéaux $\alpha$-réduits primitifs des ordres $O_{4 D}$ et $O_{D}$ :

ThÉORÈme 2. Soit $D>5$ un entier non carré congru à 1 modulo 4 .

(a) Si $D \equiv 5(\bmod 8)$ alors $2 N^{*}<N \leq 4 N^{*}$.

(b) Si $D \equiv 1(\bmod 8)$ alors $N^{*} / 2 \leq N<2 N^{*}$.

2. Idéaux $\alpha$-réduits. Dans tout ce travail nous poserons $\alpha=(1+\sqrt{5}) / 2$ et $\beta=(1-\sqrt{5}) / 2$. Soit $\Delta$ un discriminant positif, c'est-à-dire un entier 
positif non carré congru à 0 ou 1 modulo 4 . Les idéaux primitifs de l'ordre $O_{\Delta}$ de discriminant $\Delta$ sont les $\mathbb{Z}$-modules $I=[a,(b+\sqrt{\Delta}) / 2]$ tels que

$$
a>0, \quad\left(\Delta-b^{2}\right) /(4 a)=c \in \mathbb{Z}, \quad(a, b, c)=1 .
$$

Le nombre $a$ est la norme $N(I)$ de l'idéal $I$. La classe modulo 1 du nombre $\varphi=(b+\sqrt{\Delta}) /(2 a)$ est déterminée par $I$ et inversement le nombre $\varphi$ détermine l'idéal $I$. Nous dirons que $I$ et $\varphi$ sont associés et écrirons $I=I(\varphi)$, ce qui signifie que

$$
I=a[1, \varphi], \quad \varphi=\frac{b+\sqrt{\Delta}}{2 a}
$$

où $a$ et $b$ vérifient (2.1). Si $a$ et $b$ vérifient (2.1) nous dirons que le discriminant de $I$ et de $\varphi$ est $\Delta$. Dans toute la suite nous supposerons que $\Delta \neq 5$. Alors parmi les représentants de $\varphi$ modulo 1 il en existe un, et un seul, dont le conjugué $\bar{\varphi}$ vérifie la condition $\beta<\bar{\varphi}<\beta+1$. Soit $\varphi(I)$ ce nombre. Nous pouvons maintenant définir les idéaux et les nombres $\alpha$-réduits.

Définition 1. Le nombre $\varphi$ est $\alpha$-réduit si $\varphi>2$ et $\beta<\bar{\varphi}<\beta+1$. L'idéal $I$ est $\alpha$-réduit si le nombre $\varphi(I)$ est $\alpha$-réduit.

La caractérisation suivante des idéaux $\alpha$-réduits est très utile.

Proposition 1. L'idéal $I=a[1, \varphi]$ est $\alpha$-réduit si, et seulement si,

$$
[\varphi]-\bar{\varphi}>\alpha \text {. }
$$

Démonstration. Le nombre $[\varphi]-\bar{\varphi}$ est défini par $\varphi$ modulo 1 . Choisissons $\varphi$ de manière que $\beta<\bar{\varphi}<\beta+1$, c'est-à-dire, $1<\bar{\varphi}+\alpha<2$. On voit donc que $[\varphi]>\bar{\varphi}+\alpha$ si, et seulement si, $\varphi>2$, ce qui prouve (2.2).

Exemple. Les idéaux $O_{D}$ et $O_{4 D}$ sont $\alpha$-réduits. En effet, ces idéaux sont respectivement les $\mathbb{Z}$-modules $[1,(1+\sqrt{D}) / 2]$ et $[1, \sqrt{D}]$, et leurs nombres $\varphi$ associés, respectivement $(1+\sqrt{D}) / 2$ et $\sqrt{D}$, vérifient $(2.2)$.

Nous comparons maintenant les idéaux $\alpha$-réduits avec les idéaux réduits au sens usuel et avec les idéaux $k$-réduits introduits dans le travail [5]. Nous commençons par un lemme.

Lemme 1. Soit $\varphi=(b+\sqrt{\Delta}) /(2 a)$ un nombre irrationnel quadratique et $k \geq 0$ un entier. Alors

$$
[\varphi]-\bar{\varphi}>k \Leftrightarrow \varphi+[-\bar{\varphi}]>k .
$$

Démonstration. Les nombres $[\varphi]-\bar{\varphi}$ et $\varphi+[-\bar{\varphi}]$ ne dépendant que de $\varphi$ modulo 1 , choisissons $\varphi$ pour que $-1<\bar{\varphi}<0$, si bien que $[-\bar{\varphi}]=0$ et $k-1<k+\bar{\varphi}<k$. Alors $[\varphi]>k+\bar{\varphi}$ si, et seulement si, $[\varphi] \geq k$, c'est-à-dire si, et seulement si, $\varphi>k=k+[-\bar{\varphi}]$, ce qu'il fallait démontrer.

Rappelons que, pour $k$ entier, $k \geq 0$, les idéaux $k$-réduits ont été définis dans [5] par la condition $\varphi+[-\bar{\varphi}]>k$, condition que l'on peut remplacer 
par $[\varphi]-\bar{\varphi}>k$. De plus les idéaux réduits sont les idéaux 1-réduits. Ceci justifie la Définition 2 et prouve la Proposition 2 suivante.

DÉfinition 2. Soit $\lambda$ un nombre réel, $\lambda \geq 0$. L'idéal $I=a[1, \varphi]$ est dit $\lambda$-réduit si $[\varphi]-\bar{\varphi}>\lambda$.

Proposition 2. (a) Les idéaux $\alpha$-réduits sont réduits. Les idéaux 2réduits sont $\alpha$-réduits.

(b) Le nombre des idéaux $\alpha$-réduits est fini.

Nous aurons besoin plus tard du lemme suivant.

Lemme 2. Soit $\varphi$ un nombre $\alpha$-réduit. Alors, pour $k$ entier $\geq 2$, on a

$$
\varphi>k \Leftrightarrow[\varphi]-\bar{\varphi}>\alpha+k-2 .
$$

En effet, si $\varphi>k$ alors $[\varphi]-\bar{\varphi} \geq k-\bar{\varphi}>k-(1+\beta)=k-2+\alpha$. Réciproquement, si $[\varphi]-\bar{\varphi}>\alpha+k-2$, alors $[\varphi]>\beta+\alpha+k-2$, d'où $[\varphi] \geq k$, donc $\varphi>k$, ce qu'il fallait prouver.

3. Développement en fraction continue à l'entier le plus proche. Soit $I$ un idéal primitif de $O_{\Delta}$ défini par un nombre $\varphi$. Le $\alpha$-successeur $\varrho(\varphi)$ de $\varphi$ et celui $\varrho(I)$ de $I$ sont définis par

$$
\left\{\begin{array}{l}
\varphi=q+\frac{\varepsilon}{\varrho(\varphi)}, \quad \varrho(\varphi)>2, q \in \mathbb{Z}, \varepsilon= \pm 1, \\
\varrho(I)=I(\varrho(\varphi)) .
\end{array}\right.
$$

LEMme 3. On a

$$
\varrho(I)=\left|\frac{1}{\overline{\varrho(\varphi)}}\right| I .
$$

Dém on stratio n. Il suffit d'appliquer la Proposition 3 de [9] à (3.1).

Proposition 3. (a) Si l'idéal I ou le nombre $\varphi$ est $\alpha$-réduit alors $\varrho(\varphi)$ est $\alpha$-réduit et $\varepsilon \overline{\varrho(\varphi)}<0$.

(b) Si le nombre $\varphi$ est $\alpha$-réduit il existe un, et un seul, nombre $\alpha$-réduit $\psi$ tel que $\varphi=\varrho(\psi)$.

Démonstration. Posant $\varphi^{\prime}=\varrho(\varphi)$ il suffit de vérifier que $\beta<\overline{\varphi^{\prime}}<$ $\beta+1$ et $\varepsilon \overline{\varphi^{\prime}}<0$. Comme $\varphi>2$ on a $q \geq 2$, et même $q \geq 3$ si $\varepsilon=-1$, d'où

$$
\begin{array}{ll}
0>\overline{\varphi^{\prime}}=\frac{1}{\bar{\varphi}-q}>\frac{1}{\beta+1-2}=\beta, & \text { si } \varepsilon=+1, \\
0<\overline{\varphi^{\prime}}=\frac{1}{q-\bar{\varphi}}<\frac{1}{3-(\beta+1)}=\beta+1, & \text { si } \varepsilon=-1,
\end{array}
$$

ce qui prouve (a).

Pour prouver (b) posons $\psi=q+\varepsilon / \varphi$. Il s'agit de montrer que $q \in \mathbb{Z}$ et $\varepsilon= \pm 1$ sont bien déterminés par les conditions $\beta<\bar{\psi}<\beta+1$ et $\psi>2$. Comme $\beta<\bar{\varphi}<\beta+1$ on voit que $1 / \bar{\varphi}<-\alpha$ si $\bar{\varphi}<0$, et $1 / \bar{\varphi}>\alpha+1$ si 
$\bar{\varphi}>0$. La condition $\beta<q+\varepsilon / \bar{\varphi}<\beta+1$ avec $q \geq 2$ montre que $\varepsilon \bar{\varphi}<0$, ce qui détermine $\varepsilon$ et donc $q$. Il reste à prouver que $\psi>2$. Si $\bar{\varphi}>0$ alors $\varepsilon=-1$ et $q>\beta+1 / \bar{\varphi}>\beta+\alpha+1=2$, donc $q \geq 3$ et $\psi>2$. Si $\bar{\varphi}<0$ alors $\varepsilon=+1$ et $q>-1 / \bar{\varphi}+\beta>\alpha+\beta=1$, donc $q \geq 2$ et $\psi>2$. Ceci achève de prouver la Proposition 3.

Corollaire 1. L'application $\varrho: \varphi \rightarrow \varphi^{\prime}$ définie par (3.1) définit une bijection sur l'ensemble des idéaux $\alpha$-réduits de discriminant $\Delta$.

Démonstration. Comme $\varphi=q+\varepsilon / \varphi^{\prime}$, les nombres $\varphi$ et $\varphi^{\prime}$ sont équivalents, donc les idéaux $I(\varphi)$ et $I\left(\varphi^{\prime}\right)$ sont des idéaux équivalents de même discriminant. Les Propositions 2 et 3 montrent alors que l'application $\varrho$ est une injection de l'ensemble fini des idéaux $\alpha$-réduits dans lui-même, donc une bijection. Ceci prouve le Corollaire 1.

Nous considérons maintenant la suite des nombres $\varphi_{n}=\varrho^{n}\left(\varphi_{0}\right)$ définis par (1.2) et (3.1) et la suite de leurs idéaux associés $I_{n}=I\left(\varphi_{n}\right)$. Comme $\varphi_{n}(n \geq 1)$ et $I_{n}(n \geq 0)$ ne dépendent que de $I=I_{0}=I(\varphi)$ nous pouvons supposer $\varphi_{0}>2$. Alors, pour tout $n \geq 0$, on a

$$
\varphi_{n}>2, \quad q_{n} \geq 2, \quad q_{n} \geq 3 \quad \text { si } \varepsilon_{n}=-1 .
$$

Nous définissons trois suites d'entiers $A_{n}, B_{n}(n \geq-1)$ et $\mu_{n}(n \geq 0)$ par

$$
\begin{array}{lll}
A_{-1}=1, \quad A_{0}=q_{0}, \quad A_{n}=A_{n-1} q_{n}+\varepsilon_{n-1} A_{n-2} & (n \geq 1), \\
B_{-1}=0, \quad B_{0}=1, \quad B_{n}=B_{n-1} q_{n}+\varepsilon_{n-1} B_{n-2} & (n \geq 1), \\
\mu_{0}=1, \quad \mu_{n}=A_{n} B_{n-1}-A_{n-1} B_{n} \quad(n \geq 1) . &
\end{array}
$$

On vérifie par récurrence sur $n$ l'exactitude des relations suivantes :

$$
\begin{gathered}
\varphi_{0}=\frac{A_{n-1} \varphi_{n}+\varepsilon_{n-1} A_{n-2}}{B_{n-1} \varphi_{n}+\varepsilon_{n-1} B_{n-2}}, \quad \varphi_{n}=\varepsilon_{n-1} \frac{A_{n-2}-\varphi_{0} B_{n-2}}{B_{n-1} \varphi_{0}-A_{n-1}} \quad(n \geq 1), \\
\mu_{n}=(-1)^{n-1} \varepsilon_{0} \ldots \varepsilon_{n-1} \quad(n \geq 0) \\
\varphi_{1} \ldots \varphi_{n}=B_{n-1} \varphi_{n}+\varepsilon_{n-1} B_{n-2} \quad(n \geq 1) .
\end{gathered}
$$

Comme $q_{n}(n \geq 1)$ et $\varepsilon_{n}$ ne dépendent que de la classe de $\varphi$ modulo 1 , c'est-à-dire de $I=I(\varphi)$, les suites $B_{n}, A_{n}$ et $\mu_{n}$ ne dépendent que de l'idéal $I$.

Comme $q_{n} \geq 2$ on déduit de (3.2) et (3.3) que

$$
0<A_{n}<A_{n+1} \quad(n \geq-1), \quad 0<B_{n}<B_{n+1} \quad(n \geq 0)
$$

si bien que $A_{n}$ et $B_{n}$ tendent vers $\infty$ avec $n$.

Nous pouvons maintenant énoncer et démontrer le résultat principal de ce paragraphe.

THÉORÈme 3. (a) Il existe $n_{0}$ tel que l'idéal $I_{n}=\varrho^{n}(I)$ et le nombre $\varphi_{n}=$ $\varrho^{n}(\varphi)$ soient $\alpha$-réduits pour $n \geq n_{0}$. Les suites $I_{n}$ et $\varphi_{n}$ sont périodiques à partir de $n_{0}$. 
(b) Deux idéaux $\alpha$-réduits I et $I^{\prime}$ équivalents sont dans la même période. Plus précisément, si $I^{\prime}=\lambda I$ avec $\lambda>1$, il existe $n \geq 1$ tel que $I^{\prime}=\varrho^{n}(I)$ et $\lambda=\prod_{i=1}^{n}\left|1 / \bar{\varphi}_{i}\right|$.

Démonstration. De (3.4), (3.5) et (3.6) on déduit

$$
\frac{A_{n}}{B_{n}}-\varphi_{0}=\frac{-\mu_{n+1}}{B_{n}^{2} \varphi_{n}+\varepsilon_{n} B_{n} B_{n-1}},
$$

ce qui montre que $A_{n} / B_{n}-\varphi_{0} \rightarrow 0$ quand $n \rightarrow \infty$. Mais, d'après (3.5), on a

$$
-\varepsilon_{n} \bar{\varphi}_{n+1}=\frac{B_{n-1}}{B_{n}} \cdot \frac{\varphi_{0}-\bar{\varphi}_{0}+A_{n-1} / B_{n-1}-\varphi_{0}}{\varphi_{0}-\bar{\varphi}_{0}+A_{n} / B_{n}-\varphi_{0}},
$$

ce qui montre que $-\varepsilon_{n} \bar{\varphi}_{n+1} B_{n} / B_{n-1} \rightarrow 1$ quand $n \rightarrow \infty$. Il existe donc $n_{0}$ tel que $\varepsilon_{n} \bar{\varphi}_{n+1}<0$ pour $n \geq n_{0}$. On vérifie par récurrence que, pour $n \geq 1$,

$$
\text { si } \quad \varepsilon_{n}=-1 \quad \text { alors } \quad B_{n}>\frac{3+\sqrt{5}}{2} B_{n-1} .
$$

Alors, utilisant (3.3), on trouve, pour $n \geq 1$,

$$
\begin{array}{ll}
B_{n}>q_{n} B_{n-1} & \text { si } \varepsilon_{n-1}=1, \\
B_{n}>\left(q_{n}+\alpha-2\right) B_{n-1} & \text { si } \varepsilon_{n-1}=-1,
\end{array}
$$

ce qui montre que

$$
\left\{\begin{array}{l}
\text { si } \varepsilon_{n}=1 \text { on a } B_{n-1} / B_{n}<1 / 2, \text { sauf si } q_{n}=2, \varepsilon_{n-1}=-1, \\
\text { si } \varepsilon_{n}=-1 \text { on a } B_{n-1} / B_{n}<1 / 3, \text { sauf si } q_{n}=3, \varepsilon_{n-1}=-1 .
\end{array}\right.
$$

Supposons $n \geq n_{0}$ de sorte que $\varepsilon_{n} \bar{\varphi}_{n+1}<0$. Comme $-1 / 2>\beta$ et $1 / 3<\beta+1$ on voit que, si $\varphi_{n+1}$ n'était jamais $\alpha$-réduit, on aurait, pour tout $n$ assez grand, $\varepsilon_{n-1}=-1, \varepsilon_{n}=1, q_{n}=2$ ou bien $\varepsilon_{n-1}=\varepsilon_{n}=-1, q_{n}=3$. Le premier cas ne peut convenir, car $\varepsilon_{n}=1, \varepsilon_{n+1}= \pm 1$, donc $\varphi_{n+2}$ serait $\alpha$-réduit. Donc $\varepsilon_{n}=-1$ et $q_{n}=3$ à partir d'un certain rang. Mais alors $\varphi_{n}=(3+\sqrt{5}) / 2$ à partir de ce rang, cas exclu car $\Delta \neq 5$. Ceci montre qu'il existe $n_{0}$ tel que $\varphi_{n_{0}}$ soit $\alpha$-réduit. Le fait que la suite $\varphi_{n}$ soit périodique pour $n \geq n_{0}$ vient de ce que $\varphi_{n}=\varrho^{n-n_{0}}\left(\varphi_{n_{0}}\right)$ avec les notations du Corollaire 1. Ceci achève la démonstration de (a).

Avant de démontrer (b) remarquons que

$$
\frac{B_{n+1}}{B_{n-1}}=q_{n+1} \frac{B_{n}}{B_{n-1}}+\varepsilon_{n},
$$

ce qui, compte tenu de (3.10) et (3.11), donne, dans tous les cas,

$$
B_{n+1}>(2+\sqrt{5}) B_{n-1} .
$$

Il reste à prouver (b), c'est-à-dire que deux nombres équivalents $\alpha$-réduits sont dans la même période. Nous adaptons le raisonnement classique (voir par exemple [3], §10-6, 10-10, 10-11) à notre objet. Nous commençons par le 
Lemme 4. Si $\varphi_{0}=(P \psi+Q) /(R \psi+S)$ avec $P S-Q R= \pm 1, R>2|S|$ $>0$ et $\psi>0$, il existe $n$ tel que $\psi=\varphi_{n}, P=A_{n}, R=B_{n}, S=\varepsilon_{n-1} B_{N-1}$ et $Q=\varepsilon_{n-1} A_{N-1}$.

Démonstration. Nous appliquons le processus (3.1) au nombre rationnel $P / R=\varphi_{0}^{\prime}$. Dans ce cas (3.1) s'écrit successivement

$$
\begin{aligned}
& P=q_{0}^{\prime} R+\varepsilon_{0}^{\prime} r_{1}, \quad \varphi_{0}^{\prime}=P / R, \quad q_{0}^{\prime}, \quad 0 \leq 2 r_{1}<R, \\
& R=q_{1}^{\prime} r_{1}+\varepsilon_{1}^{\prime} r_{2}, \quad \varphi_{1}^{\prime}=R / r_{1}, \quad q_{1}^{\prime} \geq 2, \quad 0 \leq 2 r_{2}<r_{1}, \\
& \text {... } \\
& r_{n-1}=q_{n}^{\prime} r_{n}+\varepsilon_{n}^{\prime} r_{n+1}, \quad \varphi_{n}^{\prime}=r_{n-1} / r_{n}, \quad q_{n}^{\prime} \geq 2, \quad 0 \leq 2 r_{n+1}<r_{n}, \\
& \text {... } \\
& r_{N-1}=q_{N}^{\prime} r_{N}, \quad \varphi_{N}^{\prime}=r_{N-1} / r_{N}=q_{N}^{\prime}, \quad q_{N}^{\prime} \geq 2, \quad r_{N+1}=0 .
\end{aligned}
$$

Le fait qu'il existe $N$ tel que $r_{N+1}=0$ vient de ce que la suite des entiers positifs $r_{i}$ est strictement décroissante.

Si $q_{N}^{\prime}=2$ et $\varepsilon_{N-1}^{\prime}=1$ la fin du développement de $P / R$ est $q_{N-1}^{\prime}-1 / 2$ avec $q_{N-1}^{\prime} \geq 3$; ceci s'écrit aussi $\left(q_{N-1}^{\prime}-1\right)+1 / 2$, donc on peut supposer que l'on n'a pas à la fois $q_{N}^{\prime}=2, \varepsilon_{N-1}^{\prime}=-1$, et donc, d'après (3.11), que $2 B_{N-1}<B_{N}$.

Tenant compte de (3.5), (3.2) et (3.3), il vient

$$
\frac{P}{R}=\frac{A_{N-1} q_{N}^{\prime}+\varepsilon_{N-1}^{\prime} A_{N-2}}{B_{N-1} q_{N}^{\prime}+\varepsilon_{N-1}^{\prime} B_{N-2}}=\frac{A_{N}}{B_{N}},
$$

puis, comme $(P, R)=\left(A_{N}, B_{N}\right)=1, R$ et $B_{N}>0$, on voit que $P=A_{N}$ et $R=B_{N}$. Donc $P S-Q R=\varepsilon\left(P B_{N-1}-Q A_{N-1}\right)(\varepsilon= \pm 1)$, d'où résulte $P\left(S-\varepsilon B_{N-1}\right)=R\left(Q-\varepsilon A_{N-1}\right)$. Comme $(P, R)=1$ et $|S|+B_{N-1}<R$ on a $S=\varepsilon B_{N-1}, Q=\varepsilon A_{N-1}$, d'où

$$
\varepsilon_{0}=\frac{P \psi+Q}{R \psi+S}=\frac{A_{N} \psi+\varepsilon A_{N-1}}{B_{N} \psi+\varepsilon B_{N-1}}=\frac{A_{N} \varphi_{N+1}+\varepsilon_{N} A_{N-1}}{B_{N} \varphi_{N+1}+\varepsilon_{N} B_{N-1}} .
$$

Développant et tenant compte de (3.4) et (3.6), on obtient $\varepsilon \mu_{N} \psi=$ $\varepsilon_{N} \mu_{N} \varphi_{N+1}$. Comme $\psi>0$ et $\varphi_{N+1}>2$ on a donc $\psi=\varphi_{N+1}$ et $\varepsilon_{N}=\varepsilon$, ce qui achève la démonstration du Lemme 4 .

Nous pouvons maintenant achever la démonstration du Théorème 3(b).

Soient $\psi$ et $\varphi$ deux nombres $\alpha$-réduits équivalents, $\psi=(a \varphi+b) /(c \varphi+d)$ avec $a, b, c, d$ entiers tels que $a d-b c= \pm 1$, où nous pouvons supposer $c \varphi+d$ $>0$ en changeant, si nécessaire, les signes de $a, b, c$ et $d$. Développons $\varphi=$ $\varphi_{0}$ en fraction continue à l'entier le plus proche et remplaçons; il vient, d'après (3.5),

$$
\psi=\frac{P \varphi_{n}+Q}{R \varphi_{n}+S} \quad \text { avec } R=c A_{n-1}+d B_{n-1}, S=\varepsilon_{n-1}\left(c A_{n-2}-d B_{n-2}\right) .
$$


D'après (3.9) et (3.7) on a $A_{n-1}=\varphi B_{n-1} \pm 1 / \theta_{n-1}, A_{n-2}=\varphi B_{n-2} \pm 1 / \theta_{n-2}$, où on a posé $\theta_{k}=\varphi_{1} \ldots \varphi_{k}$. On a donc

$$
R=B_{n-1}(c \varphi+d) \pm \frac{c}{\theta_{n-1}}, \quad S=\varepsilon_{n-1}\left(B_{n-2}(c \varphi+d) \pm \frac{c}{\theta_{n-2}}\right) .
$$

Comme, d'après (3.13), $B_{n-1}>2 B_{n-2}$ pour une infinité d'indices $n$ et que $\theta_{n-1}$ et $\theta_{n-2} \rightarrow \infty$ quand $n \rightarrow \infty$, on voit qu'il existe $n$ tel que $R>2|S|>0$, ce qui, d'après le Lemme 4 , montre que $\varphi_{n}$ est dans la période de $\psi$. Donc $\varphi$ et $\psi$ sont dans la même période. Tenant compte du Lemme 3, ceci achève la démonstration du Théorème 3(b).

4. Définition de l'application $\psi$. Comparaison des nombres des idéaux $\alpha$-réduits des discriminants $D$ et $4 D$ pour $D \equiv 5(\bmod 8)$. Dans cette section nous désignerons par $\varphi$ les nombres de discriminant $4 D$ et par $\omega$ les nombres de discriminant $D$. Soit $E$ (respectivement $E^{*}$ ) l'ensemble des idéaux $\alpha$-réduits de discriminant $4 D$ (respectivement $D$ ). Soit $I=a[1, \varphi] \in E$ avec $\varphi=(b+\sqrt{D}) / a$, où $\varphi$ est choisi $\alpha$-réduit. Comme $D=b^{2}+a c \equiv 1(\bmod 4)$ on voit que soit $a \equiv 1(\bmod 2)$, soit $a \equiv 0$ $(\bmod 4)$ et $b \equiv 1(\bmod 2)$. Nous aurons besoin du lemme suivant.

Lemme 5. (i) Soit $\varphi=(b+\sqrt{D}) / a$ un nombre de discriminant $4 D$.

- Si $a \equiv b \equiv 1(\bmod 2)$ les nombres $\varphi / 2$ et $2 / \varphi$ sont de discriminant $D$.

- Si $a \equiv 1, b \equiv 0(\bmod 2)$ les nombres $(\varphi-1) / 2$ et $2 /(\varphi-1)$ sont de discriminant $D$.

- Si $a \equiv 0(\bmod 4)$ le nombre $2 \varphi$ est de discriminant $D$.

(ii) Soit $\omega=(b+\sqrt{D}) /(2 a)$ un nombre de discriminant $D$. Si $D \equiv$ $5(\bmod 8)$ les nombres $2 \omega, \omega / 2$ et $2 / \omega$ sont de discriminant $D$.

Démonstration. (i) Si $b \equiv 0(\bmod 2)$ on remplace $b$ par $b-a$. Alors $D=b^{2}+a c$ s'écrit $D=b^{2}+a\left(4 c^{\prime}\right)$ avec $\left(a, b, c^{\prime}\right)=1$ si $a \equiv 1(\bmod 2)$, et $D=b^{2}+\left(4 a^{\prime}\right) c$ avec $\left(a^{\prime}, b, c\right)=1$ si $a=4 a^{\prime}$. Tenant compte de ce que les nombres $\lambda$ et $1 / \lambda$ sont équivalents ceci, prouve (i).

(ii) Quand $D \equiv 5(\bmod 8)$ l'égalité $D=b^{2}+4 a c$ montre que $a \equiv c \equiv 1$ $(\bmod 2)$. Comme on a $(a, b, c)=1$ on voit que

$$
\begin{aligned}
& 2 \omega=\frac{2 b+\sqrt{4 D}}{2 a}, \quad 4 D=(2 b)^{2}+4 a(4 c) \quad \text { avec }(a, 2 b, 4 c)=1, \\
& \frac{\omega}{2}=\frac{2 b+\sqrt{4 D}}{2 \cdot 4 a}, \quad 4 D=(2 b)+4(4 a) c \quad \text { avec }(4 a, 2 b, c)=1,
\end{aligned}
$$

ce qui prouve que $2 \omega, \omega / 2$, et aussi $2 / \omega$, sont de discriminant $4 D$ et prouve (ii). 
Nous définissons une partition de $E$ en cinq sous-ensembles $E_{1}, E_{1}^{\prime}, E_{2}$, $E_{2}^{\prime}$ et $E_{4}$ et une application $\psi$ de $E$ dans $E^{*}$ définie par ses restrictions à chacun de ces sous-ensembles.

$$
\left\{\begin{array}{lc}
E_{1}: a \equiv b \equiv 1(\bmod 2), \bar{\varphi}<0, & \psi(I)=I(2 / \varphi), \\
E_{1}^{\prime}: a \equiv b \equiv 1(\bmod 2), \bar{\varphi}>0, & \psi(I)=I(-2 / \varphi), \\
E_{2}: a \equiv 1, b \equiv 0(\bmod 2), \varphi>3 \text { et } \bar{\varphi}<2-\sqrt{5}, & \psi(I)=I((\varphi-1) / 2), \\
E_{2}^{\prime}: a \equiv 1, b \equiv 0(\bmod 2), \varphi<3 \text { ou } \bar{\varphi}>2-\sqrt{5}, \\
\\
E_{4}: a \equiv 0(\bmod 4), & \psi(I)=I(2 /(\varphi-1)),
\end{array}\right.
$$

Nous désignerons par $\psi_{1}, \psi_{1}^{\prime}, \psi_{2}, \psi_{2}^{\prime}$ et $\psi_{4}$ les restrictions de $\psi$ à $E_{1}, E_{1}^{\prime}$, $E_{2}, E_{2}^{\prime}$ et $E_{4}$ respectivement. Remarquons que l'on a

$$
\begin{gathered}
\alpha=\frac{1+\sqrt{5}}{2}<\sqrt{5}<\alpha+1<2 \alpha<\alpha+2<\alpha+3, \\
\beta=\frac{1-\sqrt{5}}{2}<2-\sqrt{5}<0<\frac{3-\sqrt{5}}{4}<\beta+1=\frac{3-\sqrt{5}}{2} .
\end{gathered}
$$

Avec les définitions (4.1) nous avons

Proposition 4. L'idéal $\psi(I)$ est un idéal de discriminant D. De plus, pour $D \equiv 5(\bmod 8)$,

(1) $\psi_{1}$ est une bijection de $E_{1}$ sur les idéaux $(1+\sqrt{5})$-réduits de $E^{*}$,

(2) $\psi_{1}^{\prime}$ est une bijection de $E_{1}^{\prime}$ sur les idéaux $(2+\sqrt{5})$-réduits de $E^{*}$,

(3) $\psi_{2}$ est une bijection de $E_{2}$ sur les idéaux de $E^{*}$ tels que $\bar{\omega}>(3-\sqrt{5}) / 4$,

(4) l'image de $E_{2}^{\prime}$ par $\psi_{2}^{\prime}$ est formée des idéaux $\alpha$-réduits non $(2+\sqrt{5})$ réduits de $E^{*}$, l'image réciproque d'un idéal de $E^{*}$ est formée de deux éléments si $\sqrt{5}<[\omega]-\bar{\omega}<1+\sqrt{5}$, un seul sinon,

(5) l'image de $E_{4}$ par $\psi_{4}$ est formée des idéaux $2 \alpha$-réduits de $E^{*}$, l'image réciproque d'un idéal de $E^{*}$ par $\psi_{4}$ est formée d'un idéal si $2 \alpha<[\omega]-\bar{\omega}<$ $2+\alpha$ et $\bar{\omega}<2-\sqrt{5}$ ou $2+\alpha<[\omega]-\bar{\omega}<3+\alpha$ et $\bar{\omega}>2-\sqrt{5}$, et de deux idéaux si $2+\alpha<[\omega]-\bar{\omega}<3+\alpha$ et $\bar{\omega}<2-\sqrt{5}$ ou $3+\alpha<[\omega]-\bar{\omega}$.

Dém onstration. Le Lemme 5(i) montre que, dans tous les cas, l'idéal $\psi(I)$ est de discriminant $D$. Il reste à vérifier les points 1 à 5 . Pour cela nous partons d'un idéal $I=a[1, \varphi]$, où $\varphi$ est $\alpha$-réduit appartenant successivement à $E_{1}, E_{1}^{\prime}, E_{2}, E_{2}^{\prime}, E_{4}$, et étudions son image par $\psi$. Puis, partant d'un idéal $J=a[1, \omega] \in E^{*}$, où $\omega$ est $\alpha$-réduit, nous déterminons le nombre des éléments de $\psi^{-1}(J)$ dans chacun des sous-ensembles $E_{1}, E_{1}^{\prime}, E_{2}, E_{2}^{\prime}$ et $E_{4}$. Dans chacun des cinq cas le Lemme 5(ii) montre que les images inverses sont bien des idéaux de discriminant $4 D$. 
(1) Comme $\varphi$ est $\alpha$-réduit et $\bar{\varphi}<0$ on a $[2 / \varphi]-2 / \bar{\varphi}>0-2 / \beta=2 \alpha$, ce qui prouve que $\psi_{1}(I)$ est $2 \alpha$-réduit.

Réciproquement, soit $J$ un idéal $2 \alpha$-réduit de $E^{*}$. Les nombres $\varphi=\varphi(I)$ $\alpha$-réduits tels que $J=\psi_{1}(I)$ sont à chercher parmi les nombres $\varphi$ tel qu'il existe $n \in \mathbb{Z}$ tel que $\omega=2 / \varphi+n$, c'est-à-dire $\varphi=2 /(\omega-n)$, avec $\varphi>2$ et $\beta<\bar{\varphi}<\beta+1$, c'est-à-dire $n=[\omega]$ et $\beta<2 /(\bar{\omega}-[\omega])<\beta+1$. Comme $\bar{\omega}-[\omega]<0$ cette dernière condition équivaut à $[\omega]-\bar{\omega}>-2 / \beta=2 \alpha$. Ainsi pour tout $J \in E^{*}$ qui est $2 \alpha$-réduit il existe un et un seul $I \in E_{1}$ tel que $\psi_{1}(I)=J$, ce qui prouve (1).

(2) Comme $\varphi$ est $\alpha$-réduit et $0<\bar{\varphi}<\beta+1$ on a $[-2 / \varphi]+2 / \bar{\varphi}>$ $-1+2 /(1+\beta)=2+\sqrt{5}$, ce qui prouve que $\psi_{1}^{\prime}(I)$ est $(2+\sqrt{5})$-réduit.

Si $J$ est un idéal $(2+\sqrt{5})$-réduit les nombres $\varphi=\varphi(I)$ tels que $J=\psi_{1}^{\prime}(I)$ sont à chercher parmi les nombres $2 /(n-\omega), n \in \mathbb{Z}$, tels que $2 /(n-\omega)>2$, d'où $n=[\omega]+1$ et alors $\beta<2 /([\omega]+1-\bar{\omega})<\beta+1$. Comme $[\omega]+1-\bar{\omega}>0$ cette condition équivaut à $[\omega]+1-\bar{\omega}>2 /(\beta+1)=3+\sqrt{5}$ soit $[\omega]-\bar{\omega}>$ $2+\sqrt{5}$, ce qui prouve $(2)$.

(3) Si $a \equiv 1, b \equiv 0(\bmod 2), \varphi>3$ et $\bar{\varphi}<2-\sqrt{5}$ on a

$$
\left[\frac{\varphi-1}{2}\right]+\frac{1-\bar{\varphi}}{2}>1+\frac{\sqrt{5}-1}{2}=\alpha,
$$

donc $\psi_{2}(I)$ est $\alpha$-réduit.

Réciproquement, soit $J(\omega) \in E^{*}$. Les idéaux $I(\varphi)$ tels que $\psi_{2}(I)=J$ sont tels que $\omega=(\varphi-1) / 2+n, n \in \mathbb{Z}, \varphi>3, \beta<\bar{\varphi}<2-\sqrt{5}$, c'est-à-dire $\varphi=1+2(\omega-n)>3, \beta=(1-\sqrt{5}) / 2<1+2(\bar{\omega}-n)<2-\sqrt{5}$, ou bien $\omega>n+1,(-1-\sqrt{5}) / 4<\bar{\omega}-n<\beta$, ce qui montre que $n=1$ et qu'il y a une possibilité si, et seulement si, $\bar{\omega}>(3-\sqrt{5}) / 4$, ce qu'il fallait prouver.

(4) $\operatorname{Si} a \equiv 1, b \equiv 0(\bmod 2)$ et $\varphi<3$ ou $\bar{\varphi}>2-\sqrt{5}$ on a

$$
\begin{aligned}
& {\left[\frac{2}{\varphi-1}\right]+\frac{2}{1-\bar{\varphi}}>1+\frac{2}{1-\beta}=\sqrt{5}>\alpha \quad \text { si } \varphi<3,} \\
& {\left[\frac{2}{\varphi-1}\right]+\frac{2}{1-\bar{\varphi}}>0+\frac{2}{-1+\sqrt{5}}=\alpha \quad \text { si } \bar{\varphi}>2-\sqrt{5},}
\end{aligned}
$$

ce qui prouve que $\psi_{2}^{\prime}(I)$ est $\alpha$-réduit.

L'idéal $J=J(\omega) \in E^{*}$ étant donné, les nombres $\alpha$-réduits $\varphi=\varphi(I)$ tels que $J=\psi_{2}^{\prime}(I)$ sont à chercher parmi les nombres tels que $\omega-n=2 /(\varphi-1)$, $n \in \mathbb{Z}$, c'est-à-dire $\varphi=1+2 /(\omega-n)$ vérifiant en outre $\varphi<3$ ou $\bar{\varphi}>2-\sqrt{5}$, c'est-à-dire soit

$$
1<\frac{2}{\omega-n}<2 \quad \text { et } \quad \beta-1<\frac{2}{\bar{\omega}-n}<\beta,
$$

soit

$$
2<\frac{2}{\omega-n} \quad \text { et } \quad 1-\sqrt{5}<\frac{2}{\bar{\omega}-n}<\beta .
$$


La première alternative impose $1<\omega-n<2$, c'est-à-dire $n=[\omega]-1$ et la deuxième condition s'écrit $\sqrt{5}<[\omega]-\bar{\omega}<2+\sqrt{5}$. La deuxième alternative impose $0<\omega-n<1$, c'est-à-dire $n=[\omega]$ et la deuxième condition s'écrit $\alpha<[\omega]-\bar{\omega}<1+\sqrt{5}$. Ceci prouve (4).

(5) Si $a \equiv 0(\bmod 4)$ on a $[2 \varphi]-2 \bar{\varphi} \geq 2[\varphi]-2 \bar{\varphi}>2 \alpha$, ce qui prouve que $\psi_{4}(I)$ est $2 \alpha$-réduit. Réciproquement, soit $J$ un idéal $2 \alpha$-réduit. Par $\psi_{4}$ il peut provenir soit de $I_{1}=I(\omega / 2)$, soit de $I_{2}=I((\omega-1) / 2)$.

L'idéal $I_{1}$ est $\alpha$-réduit si, et seulement si, $[\omega / 2]-\bar{\omega} / 2>\alpha$, c'est-à-dire si, et seulement si, $2[\omega / 2]>2 \alpha+\bar{\omega}$. Comme $\beta<\bar{\omega}<\beta+1$ et $\alpha+\beta=1$ on voit que $2<1+\alpha<2 \alpha+\bar{\omega}<2+\alpha<4$, ce qui montre que $I_{1}$ est $\alpha$-réduit si, et seulement si, $[\omega / 2] \geq 2$, c'est-à-dire $\omega>4$. Appliquant le Lemme 2 , on voit que $I_{1}$ est $\alpha$-réduit si, et seulement si, $J$ est $\alpha+2$ réduit.

L'idéal $I_{2}$ est $\alpha$-réduit si, et seulement si, $\left.[(\omega-1) / 2)\right]-(\bar{\omega}-1) / 2>\alpha$, c'est-à-dire si, et seulement si, $2[(\omega-1) / 2]>2 \alpha-1+\bar{\omega}$. Ici on a $1<$ $2 \alpha-1+\bar{\omega}<3$ et donc $I_{2}$ est $\alpha$-réduit si, et seulement si, soit $\omega>3$ et $\bar{\omega}<2-\sqrt{5}$, soit $\omega>5$, ce qui, tenant compte du Lemme 2 , prouve (5) et achève la démonstration de la Proposition 4.

Corollaire 2. Soit $n_{1}(J), n_{2}(J), n_{3}(J)$ définis par

$$
\begin{gathered}
n_{1}(J)=\left\{\begin{array}{l}
1 \quad \text { si } \alpha<[\omega]-\bar{\omega}<\sqrt{5}, \\
2 \quad \text { si } \sqrt{5}<[\omega]-\bar{\omega}<2+\alpha, \\
3 \quad \text { si } 2+\alpha<[\omega]-\bar{\omega}<3+\alpha, \\
4 \quad \text { si } 3+\alpha<[\omega]-\bar{\omega} ;
\end{array}\right. \\
n_{2}(J)=1 \quad \text { si } 2 \alpha<[\omega]-\bar{\omega}<3+\alpha \text { et } \bar{\omega}<2-\sqrt{5}, \quad n_{2}(J)=0 \quad \text { sinon; } \\
n_{3}(J)=1 \quad \text { si } \bar{\omega}>(3-\sqrt{5}) / 4, \quad n_{3}(J)=0 \quad \text { sinon. }
\end{gathered}
$$

Alors $\operatorname{card}\left(\psi^{-1}(J)\right)=n_{1}(J)+n_{2}(J)+n_{3}(J)$.

Corollaire 3. Parmi les $N^{*}$ idéaux $\alpha$-réduits de discriminant $D \equiv 5$ (mod 8) désignons par

- $N_{1}^{*}$ le nombre des idéaux $\alpha$-réduits non $\sqrt{5}$-réduits,

- $N_{2}^{*}$ le nombre des idéaux $\sqrt{5}$-réduits non $(\alpha+2)$-réduits,

- $N_{3}^{*}$ le nombre des idéaux $(\alpha+2)$-réduits non $(\alpha+3)$-réduits,

- $N_{4}^{*}$ le nombre des idéaux $(\alpha+3)$-réduits,

- $N^{\prime *}$ le nombre des idéaux $2 \alpha$ réduits non $(3+\alpha)$-réduits et tels que $\bar{\omega}<2-\sqrt{5}$,

- $N^{\prime \prime *}$ le nombre des idéaux $\alpha$-réduits tels que $\bar{\omega}>(3-\sqrt{5}) / 4$.

Alors

$$
N=\operatorname{card}(E)=N_{1}^{*}+2 N_{2}^{*}+3 N_{3}^{*}+4 N_{4}^{*}+N^{\prime *}+N^{\prime \prime *} .
$$

Démonstration des Corollaires 2 et 3. D'après (1), (2), (4) de la Proposition 4 on obtient par les applications $\psi_{1}, \psi_{1}^{\prime}$ et $\psi_{2}^{\prime}$ une fois les 
idéaux $\alpha$-réduits non $\sqrt{5}$-réduits et deux fois les idéaux $\sqrt{5}$-réduits de $E^{*}$. Ceci étant, (3) et (5) nous permettent de terminer les démonstrations.

5. L'application $\theta$ et la démonstration du Théorème 1(a). Nous désignerons par $H(4 D)$ et $H(D)$ les groupes de classes d'idéaux primitifs de discriminant $4 D$ et $D$ respectivement, et par $\varepsilon_{4 D}$ et $\varepsilon_{D}$ les unités fondamentales $>1$ des anneaux $O_{4 D}$ et $O_{D}$ respectivement. Nous désignerons aussi par $C(I)$ la classe de l'idéal $I$.

Soit $C \in H(4 D)$ et $I=[a, b+\sqrt{D}]$ un idéal de $C$. Comme $D \equiv 1(\bmod 4)$ on a $b \equiv 1(\bmod 2)$ si $a \equiv 0(\bmod 4)$ ou bien $b \not \equiv b+a(\bmod 2)$, donc on peut toujours supposer $b \equiv 1(\bmod 2)$. Alors on sait (voir par exemple [9], Théorème 1 et Corollaire 4) que l'idéal

$$
\theta(I)=\left\{\begin{array}{l}
{\left[a, \frac{b+\sqrt{D}}{2}\right] \quad \text { si } a \equiv 1(\bmod 2),} \\
{\left[\frac{a}{4}, \frac{b+\sqrt{D}}{2}\right] \quad \text { si } a \equiv 0(\bmod 4),}
\end{array}\right.
$$

est un idéal primitif de $O_{D}$ dont la classe

$$
\theta(C)=C(\theta(I))
$$

ne dépend que de la classe $C$ de $I$, et que si les idéaux $I=[a, b+\sqrt{D}]$ et $I^{\prime}=\left[a^{\prime}, b^{\prime}+\sqrt{D}\right]$, vérifiant $a \equiv a^{\prime} \equiv 1(\bmod 2)$, sont équivalents alors

$$
\text { pour tout } \lambda \in Q(\sqrt{D}) \text { tel que } I^{\prime}=\lambda I \text { on a } \theta\left(I^{\prime}\right)=\lambda \theta(I) \text {. }
$$

D'autre part, on sait (voir [2], §256, VI ou [10], §151) que l'application de $H(4 D)$ dans $H(D)$ définie par $C \rightarrow \theta(C)$ est un homomorphisme surjectif et que

$$
\left\{\begin{aligned}
\operatorname{card}(\operatorname{Ker} \theta) & =1 \Leftrightarrow \\
(1.1) & \Leftrightarrow \text { a des solutions impaires ou } D \equiv 1(\bmod 8), \\
\operatorname{card}(\operatorname{Ker} \theta) & =3 \Leftrightarrow \\
(1.1) & \text { n'a pas de solution impaire et } D \equiv 5(\bmod 8),
\end{aligned}\right.
$$

et

$$
\left\{\begin{array}{l}
(1.1) \text { a des solutions impaires } \Leftrightarrow \varepsilon_{4 D}=\varepsilon_{D}^{3}, \\
\text { (1.1) n'a pas de solution impaire } \Leftrightarrow \varepsilon_{4 D}=\varepsilon_{D} .
\end{array}\right.
$$

Les définitions (4.1), (5.1) et (5.2) de $\psi$ et $\theta$ montrent que l'application $\psi$ est compatible avec $\theta$ en ce sens que

$$
C(\psi(I))=\theta(C(I)) .
$$

Pour toute classe $C \in H(4 D)$ nous désignerons par $L(C)$ le nombre des idéaux primitifs $\alpha$-réduits de $C$, c'est-à-dire la longueur de la période de $C$, et par $L^{*}(C)$ la longueur de la période de $\theta(C)$.

Pour démontrer le Théorème 1(a) nous utiliserons le résultat suivant : 
Proposition 5. Soit $D \equiv 5(\bmod 8)$. $\geq 3$.

(a) Pour tout $J \in E^{*}$ tel que $\operatorname{card}\left(\psi^{-1}(J)\right)=1$ on a $\operatorname{card}\left(\psi^{-1}\left(\varrho^{-1}(J)\right)\right)$

(b) Soit $J \in E^{*}$ tel que card $\left(\psi^{-1}(J)\right)=5$. Il existe un entier $n_{0} \geq 1$ tel que $\operatorname{card}\left(\psi^{-1}\left(\varrho^{-n_{0}}(J)\right)\right) \leq 3$ et $\operatorname{card}\left(\psi^{-1}\left(\varrho^{-n}(J)\right)\right)=4$ pour $0<n<n_{0}$.

Dém o n stration. Nous désignerons par $\omega_{1}$ le nombre associé à $\varrho^{-1}(J)$ de sorte que il existe $q_{1}$ entier $\geq 2$ et $\varepsilon_{1}=+1$ ou -1 tel que

$$
\omega_{1}=q_{1}+\frac{\varepsilon_{1}}{\omega}, \quad \bar{\omega}_{1}=q_{1}+\frac{\varepsilon_{1}}{\bar{\omega}}, \quad \varepsilon_{1} \bar{\omega}<0
$$

(a) Si $J \in E^{*}$ est tel que card $\left(\psi^{-1}(J)\right)=1$ alors, d'après le Corollaire 2 , on a $\alpha<[\omega]-\bar{\omega}<\sqrt{5}$ et $\beta<\bar{\omega}<(3-\sqrt{5}) / 4$, ce qui montre que $1<[\omega]<\sqrt{5}+(3-\sqrt{5}) / 4<3$, d'où $[\omega]=2$ et donc

$$
2-\sqrt{5}<\bar{\omega}<\frac{3-\sqrt{5}}{4}
$$

Tenant compte de (5.7) et (5.8), on obtient

$$
\left[\omega_{1}\right]-\bar{\omega}_{1}= \begin{cases}0-\frac{1}{\bar{\omega}}>\frac{1}{\sqrt{5}-2}=2+\sqrt{5} & \text { si } \bar{\omega}<0, \\ -1+\frac{1}{\bar{\omega}}>-1+\frac{4}{3-\sqrt{5}}=2+\sqrt{5} & \text { si } \bar{\omega}>0,\end{cases}
$$

ce qui, d'après le Corollaire 2, prouve (a).

(b) Soit $J \in E^{*}$ tel que card $\left(\psi^{-1}(J)\right)=5$. D'après le Corollaire 2 on a

$$
\omega>4, \quad \frac{3-\sqrt{5}}{4}<\bar{\omega}<\frac{3-\sqrt{5}}{2} .
$$

Comme $\bar{\omega}>0$ on déduit de $(5.9)$ que $\left[\omega_{1}\right]-\bar{\omega}_{1}=-1+1 / \bar{\omega}$, d'où, tenant compte de (5.10), $\alpha<\left[\omega_{1}\right]-\bar{\omega}_{1}<2+\sqrt{5}$. Comme $\alpha+2<2+\sqrt{5}<$ $\alpha+3$, ou bien $\left[\omega_{1}\right]-\bar{\omega}_{1}<\alpha+2$ et alors $\operatorname{card}\left(\psi^{-1}\left(\varrho^{-1}(J)\right)\right) \leq 3$, ou bien $\alpha+2<\left[\omega_{1}\right]-\bar{\omega}_{1}<2+\sqrt{5}$ et alors, d'après le Lemme 2 , on a $\left[\omega_{1}\right]=4$, d'où $\bar{\omega}_{1}>2-\sqrt{5}$, de sorte que $\operatorname{card}\left(\psi^{-1}\left(\varrho^{-1}(J)\right)\right)=4$ si, et seulement si, (5.10) est vrai pour $\omega_{1}$, et sinon $\operatorname{card}\left(\psi^{-1}\left(\varrho^{-1}(J)\right)\right) \leq 3$. Ceci permet de recommencer le raisonnement avec $\omega_{1}$ à la place de $\omega$ et montre que, en parcourant la période de $J$ en sens inverse on doit, avant de revenir à $J$, passer par un idéal $J^{\prime}=\varrho^{-n_{0}}(J)$ tel que $\operatorname{card}\left(\psi^{-1}\left(J^{\prime}\right)\right) \leq 3$, ce qui prouve (b).

La Proposition 5 nous permet de montrer les Théorèmes 1(a) et 2(a).

Démonstration des Théorèmes 1 (a) et 2(a). Supposons $D \equiv$ $5(\bmod 8)$ et soit $J \in \theta(C)$ tel que $\operatorname{card}\left(\psi^{-1}(J)\right)=5$. La Proposition $5(\mathrm{~b})$ montre que $J^{\prime}=\varrho^{-n_{0}}(J)$ vérifie $\operatorname{card}\left(\psi^{-1}\left(J^{\prime}\right)\right) \leq 3$ et que à deux idéaux $J_{1}$ et $J_{2}$ distincts tels que $\operatorname{card}\left(\psi^{-1}\left(J_{1}\right)\right)=\operatorname{card}\left(\psi^{-1}\left(J_{2}\right)\right)=5$ correspondent deux idéaux $J_{1}^{\prime}$ et $J_{2}^{\prime}$ distincts. Donc, pour toute classe $C$, on a

$$
L(C) \leq 4 L^{*}(C) .
$$


Supposons maintenant que (1.1) a des solutions impaires. Alors d'après (5.4) l'application $\theta$ est bijective de sorte que, d'après (5.5), l'image par $\psi$ de la période des idéaux $\alpha$-réduits d'une classe $C$ est l'ensemble des idéaux $\alpha$-réduits de la classe $\theta(C)$. Comme à tout idéal $J \in \theta(C)$ tel que $\operatorname{card}\left(\psi^{-1}(J)\right)=1$ correspond l'idéal $J^{\prime}=\varrho^{-1}(J)$ tel que $\operatorname{card}\left(\psi^{-1}\left(J^{\prime}\right)\right) \geq 3$ on voit que l'on a, pour toute classe $C \in E$,

$$
L(C) \geq 2 L^{*}(C) .
$$

La classe principale contient l'idéal $O_{D}=J_{0}=I((1+\sqrt{D}) / 2)$ et on a

$$
\left[\frac{1+\sqrt{D}}{2}\right]-\frac{1-\sqrt{D}}{2}>\alpha+3 \text { pour } D \geq 29
$$

ce qui, d'après le Corollaire 2 , montre que $\operatorname{card}\left(\psi^{-1}\left(J_{0}\right)\right) \geq 4$, et prouve que $L_{0}>2 L_{0}^{*}$ pour $D \geq 29$, et on vérifie que $L_{0}>2 L_{0}^{*}$ est vrai pour $D=13$ et $D=21$. Ceci achève la démonstration du Théorème $1(\mathrm{a})$.

Le même raisonnement appliqué aux ensembles $E$ et $E^{*}$ prouve le Théorème $2(\mathrm{a})$.

6. Monotonie de l'application $\psi$ et démonstration du Théorème $\mathbf{1}(\mathbf{b})$. Le point essentiel est le résultat suivant qui indique comment l'idéal $\psi(I)$ avance dans sa période quand l'idéal $\alpha$-réduit $I$ décrit la sienne.

Proposition 6. Soit I un idéal $\alpha$-réduit de discriminant $4 D$, et $\varrho(I)$ son successeur défini par (3.1). On a

$$
\begin{aligned}
& \psi(\varrho(I))=\varrho^{n}(\psi(I)) \quad \text { avec } n=0,1 \text { ou } 2, \\
& \psi\left(\varrho^{2}(I)\right)=\varrho^{m}(\psi(I)) \quad \text { avec } 1 \leq m \leq 4 .
\end{aligned}
$$

Démonstration. Soit $I \in E$ un idéal $\alpha$-réduit de discriminant $4 D, \varphi$ le nombre $\alpha$-réduit associé, $\varphi^{\prime}$ le nombre associé à $\varrho(I)$ de sorte que, tenant compte de la Proposition 3, on a

$$
\varphi=q+\frac{\varepsilon}{\varphi^{\prime}}, \quad \bar{\varphi}=q+\frac{\varepsilon}{\overline{\varphi^{\prime}}}, \quad \varepsilon= \pm 1, \quad q \in \mathbb{Z}, \quad \varepsilon \overline{\varphi^{\prime}}<0 .
$$

Posant $\varphi=(b+\sqrt{D}) / a$ et $\varphi^{\prime}=\left(b^{\prime}+\sqrt{D}\right) / a^{\prime}$, on déduit de $(6.3)$,

$$
b^{\prime}=-b+a q, \quad D=b^{2}+\varepsilon a a^{\prime} .
$$

Tenant compte de ce que $D \equiv 1(\bmod 4)$ et des parités de $a, b, q, b^{\prime}, a^{\prime}$, on vérifie que

$$
\begin{cases}I \in E_{1} \text { ou } E_{1}^{\prime}, q \text { pair } & \Rightarrow \varrho(I) \in E_{4}, \\ I \in E_{1} \text { ou } E_{1}^{\prime}, q \text { impair } & \Rightarrow \varrho(I) \in E_{2} \text { ou } E_{2}^{\prime}, \\ I \in E_{2} \text { ou } E_{2}^{\prime}, q \text { pair } & \Rightarrow \varrho(I) \in E_{2} \text { ou } E_{2}^{\prime}, \\ I \in E_{2} \text { ou } E_{2}^{\prime}, q \text { impair } & \Rightarrow \varrho(I) \in E_{4}, \\ I \in E_{4} & \Rightarrow \varrho(I) \in E_{1} \text { ou } E_{1}^{\prime} .\end{cases}
$$


Soit $\omega$ et $\omega^{\prime}$ les nombres définissant $\psi(I)$ et $\psi(\varrho(I))$ respectivement, donnés par les formules (4.1). Dans chacune des éventualités de (6.5) nous développons $\omega$ en fraction continue à l'entier le plus proche et trouvons au bout de $n$ pas ( $n=0,1$ ou 2) un nombre congru modulo 1 à $\omega^{\prime}$, définissant $\psi(\varrho(I))$. Chaque pas du développement est indiqué par une flèche.

Nous commençons par montrer qu'il est impossible d'avoir $I \in E_{2}$ et $\varrho(I) \in E_{2}$.

Si $\varrho(I) \in E_{2}$ alors $\overline{\varphi^{\prime}}<2-\sqrt{5}<0$, donc $\varepsilon=1$, d'où $q=\bar{\varphi}-1 / \overline{\varphi^{\prime}}<$ $1+\beta+2+\sqrt{5}<5$, ce qui montre que $q \leq 4$.

Si $I \in E_{2}$ et $\varrho(I) \in E_{2}$ ou $E_{2}^{\prime}$ alors $q \equiv 0(\bmod 2)$ et $\varphi>3$, donc la relation $\varphi=q+\varepsilon / \varphi^{\prime}$ avec $\varphi^{\prime}>2$ montre que $q \geq 4$.

Si $I \in E_{2}$ et $\varrho(I) \in E_{2}$ on aurait $q=4$ et $\bar{\varphi}<2-\sqrt{5}$, d'où

$$
q=4=\bar{\varphi}-1 / \overline{\varphi^{\prime}}<2-\sqrt{5}+2+\sqrt{5}=4,
$$

ce qui est impossible. Il nous reste à examiner les autres possibilités que nous regroupons en neuf cas.

(1) Cas où $I \in E_{2}, \varrho(I) \in E_{2}^{\prime} ; q \equiv 0(\bmod 2) ; \omega=(\varphi-1) / 2, \omega^{\prime}=$ $2 /\left(\varphi^{\prime}-1\right)$. On a

$$
\begin{aligned}
\omega=\frac{\varphi-1}{2}= & \frac{q}{2}+\frac{1}{2}\left(\frac{\varepsilon}{\varphi^{\prime}}-1\right)=\left\{\begin{array}{ll}
\frac{q}{2}-\frac{1}{2}\left(1-\frac{1}{\varphi^{\prime}}\right) & \text { si } \varepsilon=1 \\
\frac{q}{2}-1+\frac{1}{2}\left(1-\frac{1}{\varphi^{\prime}}\right) & \text { si } \varepsilon=-1
\end{array}\right\} \\
& \rightarrow \frac{2 \varphi^{\prime}}{\varphi^{\prime}-1}=2+\frac{2}{\varphi^{\prime}-1}=2+\omega^{\prime} ; \quad n=1 .
\end{aligned}
$$

(2) Cas où $I \in E_{2}^{\prime}, \varrho(I) \in E_{2} ; q=2$ ou 4 ;

$$
\omega=2 /(\varphi-1)=2 \varphi^{\prime}\left((q-1) \varphi^{\prime}+1\right), \quad \omega^{\prime}=\left(\varphi^{\prime}-1\right) / 2 .
$$

Si $q=2$,

$$
\frac{2 \varphi^{\prime}}{\varphi^{\prime}+1}=2-\frac{2}{\varphi^{\prime}+1} \rightarrow \frac{\varphi^{\prime}+1}{2}=\frac{\varphi^{\prime}-1}{2}+1=\omega^{\prime}+1 ; \quad n=1 .
$$

Si $q=4$,

$\frac{2 \varphi^{\prime}}{3 \varphi^{\prime}+1}=1-\frac{\varphi^{\prime}+1}{3 \varphi^{\prime}+1} \rightarrow \frac{3 \varphi^{\prime}+1}{\varphi^{\prime}+1}=3-\frac{2}{\varphi^{\prime}+1} \rightarrow \frac{\varphi^{\prime}+1}{2}=\omega^{\prime}+1 ; \quad n=2$.

(3) Cas où $I \in E_{2}, \varrho(I) \in E_{4} ; q \equiv 1(\bmod 2) ; \omega=(\varphi-1) / 2, \omega^{\prime}=2 \varphi^{\prime}$. On a

$$
\omega=\frac{\varphi-1}{2}=\frac{q-1}{2}+\frac{\varepsilon}{2 \varphi^{\prime}} \rightarrow 2 \varphi^{\prime}=\omega^{\prime} ; \quad n=1 .
$$

(4) Cas où $I \in E_{1}$ ou $E_{1}^{\prime}, \varrho(I) \in E_{2} ; q \equiv 1(\bmod 2) ; \omega= \pm 2 / \varphi$, $\omega^{\prime}=\left(\varphi^{\prime}-1\right) / 2 ; \varphi^{\prime}>3$. Comme $\overline{\varphi^{\prime}}<2-\sqrt{5}$ on a $\varepsilon=1$ et $q=\bar{\varphi}-1 / \overline{\varphi^{\prime}}<$ 
$(3-\sqrt{5}) / 2+2+\sqrt{5}<5$, donc $q=3$. D'où

$$
\begin{aligned}
\omega & =\frac{ \pm 2}{3+1 / \varphi^{\prime}}=\frac{ \pm 2 \varphi^{\prime}}{3 \varphi^{\prime}+1}= \pm 1 \mp \frac{\varphi^{\prime}+1}{3 \varphi^{\prime}+1} \rightarrow \frac{3 \varphi^{\prime}+1}{\varphi^{\prime}+1} \\
& =3-\frac{2}{\varphi^{\prime}+1} \rightarrow \frac{\varphi^{\prime}+1}{2}=\omega^{\prime}+1 ; \quad n=2 .
\end{aligned}
$$

(5) Cas où $I \in E_{2}^{\prime}, \varrho(I) \in E_{2}^{\prime} ; q \equiv 0(\bmod 2) ; \omega=2 /(\varphi-1)=$ $2 \varphi^{\prime} /\left((q-1) \varphi^{\prime}+1\right), \omega^{\prime}=2 /\left(\varphi^{\prime}-1\right)$.

Si $q \geq 4$,

$$
\omega \rightarrow \frac{(q-1) \varphi^{\prime}+1}{2 \varphi^{\prime}}=\frac{q}{2}-\frac{\varphi^{\prime}-1}{2 \varphi^{\prime}} \rightarrow \frac{2 \varphi^{\prime}}{\varphi^{\prime}-1}=2+\frac{2}{\varphi^{\prime}-1} ; \quad n=2 .
$$

Si $q=2$ alors $\varepsilon=1$. Comme $\varrho(I) \in E_{2}^{\prime}$, si $\varphi^{\prime}>3$ on a $\overline{\varphi^{\prime}}>2-\sqrt{5}=$ $-1 /(2+\sqrt{5})$, d'où

$$
\begin{aligned}
& 2=\bar{\varphi}-\frac{1}{\overline{\varphi^{\prime}}}>\frac{1-\sqrt{5}}{2}+2+\sqrt{5}>2 \text { si } 2-\sqrt{5}<\overline{\varphi^{\prime}}<0, \\
& 2=\bar{\varphi}-\frac{1}{\overline{\varphi^{\prime}}}<\frac{3-\sqrt{5}}{2} \text { si } \overline{\varphi^{\prime}}>0,
\end{aligned}
$$

ce qui est impossible et montre que, si $q=2$, alors $2<\varphi^{\prime}<3$. Donc

$\omega=\frac{2 \varphi^{\prime}}{\varphi^{\prime}+1}=1-\frac{1-\varphi^{\prime}}{1+\varphi^{\prime}} \rightarrow \frac{\varphi^{\prime}+1}{\varphi^{\prime}-1}=1+\frac{2}{\varphi^{\prime}-1}=1+\omega^{\prime} ; \quad n=1$.

(6) Cas où $I \in E_{1}$ ou $E_{1}^{\prime}, \varrho(I) \in E_{2}^{\prime} ; q \equiv 1(\bmod 2) ; \omega= \pm 2 / \varphi$, $\omega^{\prime}=2 /\left(\varphi^{\prime}-1\right)$.

Si $q \geq 5$, on a $\varphi>4$, donc

$$
\begin{aligned}
\omega & =\frac{ \pm 2}{\varphi} \rightarrow \frac{\varphi}{2}=\frac{1}{2}\left(q+\frac{\varepsilon}{\varphi^{\prime}}\right) \\
& =\frac{q+\varepsilon}{2}-\varepsilon\left(\frac{\varphi^{\prime}-1}{2 \varphi^{\prime}}\right) \rightarrow 2+\frac{2}{\varphi^{\prime}-1}=2+\omega^{\prime} ; \quad n=2 .
\end{aligned}
$$

Si $q=3$,

$$
\frac{ \pm 2}{\varphi}=\frac{ \pm 2}{3+\frac{\varepsilon}{\varphi^{\prime}}}= \pm 1 \mp \frac{\varphi^{\prime}+\varepsilon}{3 \varphi^{\prime}+\varepsilon} \rightarrow \frac{3 \varphi^{\prime}+\varepsilon}{\varphi^{\prime}+\varepsilon} .
$$

Si $\varepsilon=-1$ on a

$$
\frac{3 \varphi^{\prime}-1}{\varphi^{\prime}-1}=3+\frac{2}{\varphi^{\prime}-1}=3+\omega^{\prime} ; \quad n=1 .
$$

Si $\varepsilon=1$ on a $\varphi^{\prime}<3$, sinon $\varphi^{\prime}>3$ et $2-\sqrt{5}<\overline{\varphi^{\prime}}<0$, d'où $(1-\sqrt{5}) / 2<$ $\bar{\varphi}=3+1 \sqrt{\varphi^{\prime}}<3-2-\sqrt{5}=1-\sqrt{5}$, ce qui est impossible. Donc $\varphi^{\prime}+1>$ $2\left(\varphi^{\prime}-1\right)$ et 


$$
\frac{3 \varphi^{\prime}+1}{\varphi^{\prime}+1}=2+\frac{\varphi^{\prime}-1}{\varphi^{\prime}+1} \rightarrow \frac{\varphi^{\prime}+1}{\varphi^{\prime}-1}=1+\frac{2}{\varphi^{\prime}-1}=1+\omega^{\prime} ; \quad n=2 .
$$

(7) Cas où $I \in E_{1}$ ou $E_{1}^{\prime}, \varrho(I) \in E_{4} ; q \equiv 0(\bmod 2) ; \omega= \pm 2 / \varphi, \omega^{\prime}=2 \varphi^{\prime}$. Si $q>4$ ou $q=4$ et $\varepsilon=1$ on a

$$
\omega=\frac{ \pm 2}{\varphi}=\frac{ \pm 2}{q+\frac{\varepsilon}{\varphi^{\prime}}}=\frac{ \pm 1}{\frac{q}{2}+\frac{\varepsilon}{2 \varphi^{\prime}}} \rightarrow \frac{\varepsilon}{2 \varphi^{\prime}} \rightarrow 2 \varphi^{\prime}=\omega^{\prime} ; \quad n=2 .
$$

Si $q=4$ et $\varepsilon=-1$,

$\pm \omega=\frac{2 \varphi^{\prime}}{4 \varphi^{\prime}-1}=1-\frac{2 \varphi^{\prime}-1}{4 \varphi^{\prime}-1} \rightarrow \frac{4 \varphi^{\prime}-1}{2 \varphi^{\prime}-1}=2+\frac{1}{2 \varphi^{\prime}-1} \rightarrow 2 \varphi^{\prime}-1 ; \quad n=2$.

Si $q=2$, alors $\varepsilon=1$ et

$$
\omega=\frac{ \pm 2}{\varphi}=\frac{ \pm 2 \varphi^{\prime}}{2 \varphi^{\prime}+1}= \pm 1 \mp \frac{1}{2 \varphi^{\prime}+1} \rightarrow 2 \varphi^{\prime}+1=\omega^{\prime}+1 ; \quad n=1 .
$$

(8) Cas où $I \in E_{2}^{\prime}, \varrho(I) \in E_{4} ; q \equiv 1(\bmod 2) ; \omega= \pm 2 /(\varphi-1), \omega^{\prime}=2 \varphi^{\prime}$. Si $q>3$,

$$
\pm \omega=\frac{2}{\varphi-1}=\frac{1}{\frac{q-1}{2}+\frac{\varepsilon}{2 \varphi^{\prime}}} \rightarrow \frac{\varepsilon}{2 \varphi^{\prime}} \rightarrow 2 \varphi^{\prime}=\omega^{\prime} ; \quad n=2 .
$$

Si $q=3$,

$$
\pm \omega=\frac{2 \varphi^{\prime}}{2 \varphi^{\prime}+\varepsilon}=1-\frac{\varepsilon}{2 \varphi^{\prime}+\varepsilon} \rightarrow 2 \varphi^{\prime}+\varepsilon ; \quad n=1 .
$$

(9) Cas où $I \in E_{4}, \varrho(I) \in E_{1}$ ou $E_{1}^{\prime} ; \omega=2 \varphi, \omega^{\prime}= \pm 2 / \varphi^{\prime}$. On a

$$
\omega=2 \varphi=2 q+\frac{2 \varepsilon}{\varphi^{\prime}}=2 q \pm \varepsilon \omega^{\prime}, \quad \text { donc } n=0 .
$$

Comme dans tous les cas on a $0 \leq n \leq 2$ on voit que (6.1) et l'inégalité $m \leq 4$ de (6.2) sont vrais. D'autre part $n=0$ seulement dans le cas où $I \in E_{4}$ et alors $\varrho(I) \in E_{1}$ ou $E_{1}^{\prime}$, ce qui fait que $m \geq 1$ et achève la démonstration de la Proposition 6.

Démonstration des Théorèmes 1(b) et 2(b). Soit $C$ une classe d'idéaux de $O_{4 D}$, et $I$ un idéal $\alpha$-réduit primitif de $C$. D'après (6.4), $a=N(I)$ ou $a^{\prime}=N(\varrho(I))$ est impair, donc la période des idéaux $\alpha$-réduits de $C$ contient au moins un idéal $I_{0}$ tel que $I_{0} \notin E_{4}$. Alors (4.1) et (5.1) montrent que

$$
\psi\left(I_{0}\right)= \begin{cases}\varrho^{-1}\left(\theta\left(I_{0}\right)\right) & \text { si } I_{0} \notin E_{2}, \\ \theta\left(I_{0}\right) & \text { si } I_{0} \in E_{2} .\end{cases}
$$

On a $I_{0}=\varepsilon_{4 D} I_{0}$, donc d'après (5.3) et le Lemme $3, \psi\left(I_{0}\right)=\varepsilon_{4 D} \psi\left(I_{0}\right)$.

Supposons que (1.1) n'a pas de solution impaire. D'après (5.5) on a $\varepsilon_{D}=\varepsilon_{4 D}$, donc

$$
\psi\left(I_{0}\right)=\varepsilon_{D} \psi\left(I_{0}\right)
$$


ce qui signifie, tenant compte du Théorème 3(b), que, quand l'idéal $I$ parcourt la période de $C$ de $I_{0}$ à $I_{0}$, l'idéal $\psi(I)$ avance de $\psi\left(I_{0}\right)$ à $\psi\left(I_{0}\right)$. D'après la Proposition 6 quand l'idéal $I$ avance d'un pas l'idéal $\psi(I)$ avance d'au plus deux pas, mais quand $I$ avance de deux pas alors $\psi(I)$ avance d'au moins un pas, ce qui montre que

$$
\frac{1}{2} L^{*}(C) \leq L(C) \leq 2 L^{*}(C) .
$$

De plus, la seule possibilité pour que $L(C)=2 L^{*}(C)$ est que la période de $C$ soit formée d'idéaux successivement de types $E_{1}$ ou $E_{1}^{\prime}$ avec $q=2, E_{4}, E_{1}$ ou $E_{1}^{\prime}$ avec $q=2, E_{4}, \ldots$ Mais si l'on considère la classe principale, l'idéal $(1)=I(\sqrt{D})$ est $\alpha$-réduit de type $E_{1}$ ou $E_{1}^{\prime}$ avec $q>2$ ou de type $E_{2}$ ou $E_{2}^{\prime}$, ce qui montre que, pour la classe principale, on a $L_{0}<2 L_{0}^{*}$ et achève la démonstration du Théorème 1(b).

Si $D \equiv 1(\bmod 8)$ l'application $\theta$ est bijective, ce qui montre que le Théorème $2(\mathrm{~b})$ est une conséquence du Théorème $1(\mathrm{~b})$.

Les inégalités (5.11), (5.12) et (6.8) prouvent le résultat suivant:

ThÉORÈme 4. Soit $D>5$ un entier non carré congru à 1 modulo 4. Pour toute classe $C$ d'idéaux de $O_{4 D}$,

- si l'équation $X^{2}-D Y^{2}=4$ a des solutions impaires, $2 L^{*} \leq L \leq 4 L^{*}$,

- si l'équation $X^{2}-D Y^{2}=4$ n'a pas de solution impaire, $L^{*} / 2 \leq L$ $\leq 2 L^{*}$.

7. Comparaison des périodes réduites et $\alpha$-réduites de même discriminant. Soit $I$ un idéal primitif de $O_{\Delta}$, et $\varphi$ un nombre associé à $I$. Les successeurs de $\varphi$ et de $I$ dans la réduction usuelle sont définis par

$$
\varphi=[\varphi]+\frac{1}{\sigma(\varphi)}, \quad \sigma(\varphi)>1, \quad \sigma(I)=I(\sigma(\varphi)) .
$$

La relation entre $\varrho$ définie par (3.1) et $\sigma$ est donnée par le lemme suivant.

LEMME 6.

$$
\varrho(I)= \begin{cases}\sigma(I) & \text { si } \sigma(\varphi)>2, \\ \sigma^{2}(I) & \text { si } 1<\sigma(\varphi)<2 .\end{cases}
$$

Démonstration. La définition (7.1) de $\sigma$ montre que $\varrho(\varphi)=\sigma(\varphi)$ si $\sigma(\varphi)>2$. Si $1<\sigma(\varphi)<2$ on a $[\sigma(\varphi)]=1$ et donc $\sigma(\varphi)=1+1 / \sigma^{2}(\varphi)$, d'où

$$
\varphi=[\varphi]+\frac{1}{1+1 / \sigma^{2}(\varphi)}=[\varphi]+1-\frac{1}{\sigma^{2}(\varphi)+1}
$$

ce qui montre que $\varrho(I)=I\left(\sigma^{2}(\varphi)+1\right)=I\left(\sigma^{2}(\varphi)\right)=\sigma^{2}(I)$, et prouve le Lemme 6. 
COROllaire 4. Si l'idéal I est réduit alors

$$
\varrho(I)= \begin{cases}\sigma(I) & \text { si } \sigma(I) \text { est 2-réduit, } \\ \sigma^{2}(I) & \text { si } \sigma(I) \text { n'est pas 2-réduit. }\end{cases}
$$

Démonstration. Si l'idéal $I=I(\varphi)$ est réduit le nombre $\sigma(\varphi)$ est réduit si bien que, d'après (7.2), l'idéal $\varrho(I)$ est donné par (7.3).

Lemme 7. (a) Si l'idéal $I$ est réduit et non $\alpha$-réduit alors $\sigma(I)$ est $\alpha$ réduit.

(b) Si l'idéal $I$ est $\alpha$-réduit alors $\sigma(I)$ ou $\sigma^{2}(I)$ est $\alpha$-réduit.

Démonstration. Posant $I=I(\varphi)$ on a $\sigma(I)=I(1 /(\varphi-[\varphi]))$.

(a) Si l'idéal $I$ est réduit non $\alpha$-réduit alors $1<[\varphi]-\bar{\varphi}<\alpha$, donc

$$
\left[\frac{1}{\varphi-[\varphi]}\right]+\frac{1}{[\varphi]-\bar{\varphi}}>1+\frac{1}{\alpha}=\alpha
$$

ce qui prouve que $\sigma(I)$ est $\alpha$-réduit.

(b) Si l'idéal $I$ est $\alpha$-réduit et $\sigma(I)$ non $\alpha$-réduit on a

$$
[\varphi]-\bar{\varphi}>\alpha \text { et } 1<\left[\frac{1}{\varphi-[\varphi]}\right]+\frac{1}{[\varphi]-\bar{\varphi}}<\alpha .
$$

Comme $\alpha=1+1 / \alpha$ on voit que $[1 /(\varphi-[\varphi])]=1$ d'où, comme $1 /(\varphi-[\varphi])=$ $1+1 / \sigma^{2}(\varphi)$, on a

Comme $\sigma^{2}(\varphi)>1$ et

$$
\sigma^{2}(\varphi)=\frac{\varphi-[\varphi]}{1+[\varphi]-\varphi}
$$

$$
-\overline{\sigma^{2}(\varphi)}=\frac{[\varphi]-\bar{\varphi}}{[\varphi]-\bar{\varphi}+1}>\frac{\alpha}{\alpha+1}
$$

(puisque $[\varphi]-\bar{\varphi}>\alpha$ ) on a

$$
\left[\sigma^{2}(\varphi)\right]-\overline{\sigma^{2}(\varphi)}>1+\frac{\alpha}{\alpha+1}=\alpha
$$

ce qui prouve que $\sigma^{2}(\varphi)$ est $\alpha$-réduit et achève la démonstration du Lemme 7 .

Les Lemmes 6 et 7 permettent de donner une deuxième démonstration $\mathrm{du}$ Théorème 3(a), à partir du résultat analogue pour les idéaux réduits.

En effet, on sait qu'il existe $n_{1}$ tel que $\sigma^{n}(I)$ soit réduit pour $n \geq n_{1}$. Le Corollaire 4 montre que $\varrho^{n}(I)=\sigma^{n^{\prime}}(I)$ avec $n^{\prime} \geq n$, donc l'idéal $\varrho^{n}(I)$ est réduit pour $n \geq n_{1}$. Si $\varrho^{n}(I)$ n'était jamais $\alpha$-réduit le Corollaire 4 montre que, pour tout $n \geq n_{1}$, on a $\varrho\left(\varrho^{n}(I)\right)=\sigma^{2}\left(\varrho^{n}(I)\right)$, donc, désignant par $\varphi_{n}$ le nombre réduit associé à $\varrho^{n}(I)$, on voit, d'après $(7.2)$, que $\left[\sigma\left(\varphi_{n}\right)\right]=1$ et, comme $\varphi_{n+1}$ n'est pas $\alpha$-réduit, $\left[\varphi_{n+1}\right]=1$, ce qui montre que $\varphi_{n+1}=$ $1+1 / \varphi_{n+1}$, d'où $\varphi_{n+1}=\alpha$, ce qui est impossible. Donc il existe $n_{2}$ tel que $\varrho^{n}(I)$ est $\alpha$-réduit pour $n \geq n_{2}$, ce qui est le Théorème $3(\mathrm{a})$.

Du Lemme 7 et du Théorème 3(b) on déduit 
Proposition 7. Soit $C$ une classe d'idéaux primitifs de l'ordre $O_{\Delta}$. La période $P_{\alpha}$ des idéaux $\alpha$-réduits de $C$ s'obtient en enlevant à la période $P$ des idéaux réduits de $C$ les idéaux non $\alpha$-réduits. La période $P$ ne contient pas deux idéaux consécutifs non $\alpha$-réduits.

Démonstration. Le Lemme $7\left(\right.$ a) montre que $P \cap P_{\alpha} \neq \emptyset$, ce qui d'après le Théorème $1(\mathrm{~b})$ montre que $P_{\alpha} \subset P$. La proposition résulte alors du Lemme 7(a) et (b).

\section{Références}

[1] G. Eisenstein, Aufgaben, J. Reine Angew. Math. 27 (1844), 86-87 (Werke I, Chelsea, New York, 1975, 111-112).

[2] C. F. Gauss, Arithmetische Untersuchungen (Disquisitiones Arithmeticae), Chelsea, New York, 1965.

[3] G. H. Hardy and E. M. Wright, An Introduction to the Theory of Numbers, 5ème éd., Oxford University Press, 1989.

[4] A. Hurwitz, Über eine besondere Art der Kettenbrüchen-Entwicklung reelle Grössen, Acta Math. 12 (1889), 367-405.

[5] N. Ishii, P. Kaplan and K. S. Williams, On Eisenstein's problem, Acta Arith. 54 (1990), 323-345.

[6] P. Kaplan, Idéaux k-réduits des ordres des corps quadratiques réels, J. Math. Soc. Japan 47 (1995), 171-181.

[7] P. Kaplan et P. A. Leonard, Idéaux négativement réduits d'un corps quadratique réel et un problème d'Eisenstein, Enseign. Math. 39 (1993), 196-210.

[8] P. Kaplan and K. S. Williams, Pell's equations $X^{2}-m Y^{2}=-1,-4$ and continued fractions, J. Number Theory 23 (1986), 169-182.

[9] - - - The distance between ideals in the orders of a real quadratic field, Enseign. Math. 36 (1990), 321-358.

[10] P. G. Lejeune Dirichlet und R. Dedekind, Vorlesungen über Zahlentheorie, Chelsea, New York, 1968.

[11] Y. Mimura, On odd solutions of the equation $X^{2}-D Y^{2}=4$, dans : Proc. Sympos. Analytic Number Theory and Related Topics, Gakushuin University, Tokyo, 1992, $110-118$.

[12] H. C. Williams, Eisenstein problem and continued fractions, Utilitas Math. 37 (1990), 145-158.

Département de Mathématiques

Université de Nancy I

B.P. 239

54506 Vandœuvre-lès-Nancy Cedex, France

E-mail: kaplan@iecn.u-nancy.fr
Department of Mathematics Osaka Electro-Communication University Neyagawa, Osaka, Japon E-mail: mimura@isc.osakac.ac.jp 